\title{
ON COMPOSITION OF FOUR-SYMBOL $\delta$-CODES AND HADAMARD MATRICES
}

\author{
C. H. YANG
}

(Communicated by Andrew Odlyzko)

\begin{abstract}
It is shown that key instruments for composition of four-symbol $\delta$-codes are the Lagrange identity for polynomials, a certain type of quasisymmetric sequences (i.e., a set of normal or near normal sequences) and base sequences. The following is proved: If a set of base sequences for length $t$ and a set of normal (or near normal) sequences for length $n$ exist then four-symbol $\delta$-codes of length $(2 n+1) t$ (or $n t)$ can be composed by application of the Lagrange identity. Consequently a new infinite family of Hadamard matrices of order $4 u w$ can be constructed, where $w$ is the order of Williamson matrices and $u=(2 n+1) t$ (or $n t)$. Other related topics are also discussed.
\end{abstract}

\section{INTRODUCTION}

Turyn [T1] constructed Hadamard matrices of order 4tw from a 4-symbol $\delta$-code of length $t$ and Williamson matrices of order $w$ using Baumert-Hall units. (See formal definitions for an Hadamard matrix and others below.) He $[\mathrm{T} 1, \mathrm{~T} 2]$ used certain binary sequences for construction of 4-symbol $\delta$-codes which are defined in terms of nonperiodic auto-correlation functions (whose concept originated in optics and signal transmission problems).

A method to compose 2-symbol $\delta$-codes of length $2^{k} m n$ from 2-symbol $\delta$-codes of lengths $2 m$ and $2 n$ was found by Golay [G] for $k \geq 3$ and improved by Turyn [T1] for $k \geq 2$. It is easy to construct 4-symbol $\delta$-codes of length $2^{k} t$ from a 4-symbol $\delta$-code of length $t$, which Turyn found for $t \leq 59$ (except $49,57)$ and $t=g+1$, where $g=2^{a} 10^{b} 26^{c}$ (Golay numbers), $a, b$ and $c$ are nonnegative integers [T1, T2]. However using only the definition of autocorrelation functions to prove composition of a 4-symbol $\delta$-code of length $s t$ from a 4-symbol $\delta$-code of length $t$ for odd $s$ is more difficult. This difficulty was solved by introduction of an algebraic approach, polynomials defined on the unit circle [Y4] and the Lagrange identity for polynomials [Y1,Y2]. For $s=3,7,13$ and $2 g+1$, i.e., $s=5,9,17,21,27,33,41,53,65,81, \ldots$, it was solved using this approach in $[\mathrm{Y} 1, \mathrm{Y} 2, \mathrm{Y} 3]$.

Received by the editors October 12, 1988 and, in revised form, February 2, 1989.

1980 Mathematics Subject Classification (1985 Revision). Primary 94B60, 05B20, 62K05. 
In this paper, constructions for $s=4 n+1$, and for $s \leq 31, s=51,59$ and $s=2 g+1$ are made. The former depends on the existence of near normal sequences (a 4-symbol code) for length $4 n+1$, which exist for $n \leq 11$ (and likely to exist for all $n$ ) and the latter on the existence of normal sequences (a 3-symbol code) for length $m=(s-1) / 2$, which exist for odd $m \leq 15$, $m=25,29$ and $m=g$ (a Golay number). Therefore new cases are solved for $s=11,15,19,23,25,29,31,37,45,51$ and 59; and new constructions are made for the known $4 n+1$ and $2 g+1$ cases.

These new results also lead to construction of four complementary $(1,-1)$ sequences of length $u=r s t \quad(r=m$ above, or $2 g+1)$, or equivalently 4-symbol $\delta$-codes of length $2 u$; consequently, to construction of GoethalsSeidel (Hadamard) matrices of orders $4 u$ and $8 u w$, where $w$ is the order of Williamson matrices which exist for all $w<100$ (except 35, 39, 47, 53, 59, $65,67,70,71,73,77,83,89$ and 94) [W], $w=(q+1) / 2$, where $q$ (a prime power $) \equiv 1 \quad(\bmod 4)[\mathrm{T} 3]$, and others $($ see $[\mathrm{A}],[\mathrm{M}],[\mathrm{S}]$ and $[\mathrm{W}])$. Each new case actually leads to construction of infinitely many new matrices.

\section{Preliminaries, NOTATIONS AND DEFINITIONS}

A matrix whose every entry is either $b$ or $c$ is called a $(b, c)$-matrix. Similarly a $(b, c)$-sequence has each element $b$ or $c$. An Hadamard matrix $H_{n}=$ $\left[h_{i j}\right]$ is a square $(1,-1)$-matrix of order $n$ such that $H_{n}^{t} H_{n}=n I_{n}$, where $I_{n}$ is the identity square matrix of order $n$ and $t$ indicates the transposed matrix. In $H_{n}$, distinct column vectors $v_{i}=\left[h_{1 i}, h_{2 i}, \ldots, h_{n i}\right]$ are orthogonal, i.e., $v_{i}^{t} \cdot v_{j}=\sum_{k} h_{k i} h_{k j}=0$, for $i \neq j$; similarly distinct row vectors are also orthogonal since the matrix and its transpose commute. $H_{n}$ exists only if $n=1,2$, or $4 k$, and the converse is conjectured to be valid. Hadamard matrices $H_{4 n}$ have been constructed for all $n \leq 100$ and for infinitely many $n$. For various properties and applications of Hadamard matrices, see [A], [GS], [H], [HS], $[\mathrm{HW}]$ and $[\mathrm{K}]$ in the references.

Williamson matrices $(\mathbf{W}, \mathbf{X}, \mathbf{Y}, \mathbf{Z})$ are four square $(1,-1)$-matrices of order $w$ satisfying $\mathbf{M}^{\mathbf{t}} \mathbf{N}=\mathbf{N}^{\mathbf{t}} \mathbf{M}$ for all $\mathbf{M}$ and $\mathbf{N} \in\{\mathbf{W}, \mathbf{X}, \mathbf{Y}, \mathbf{Z}\}$, i.e., all $\mathbf{M}^{\mathbf{t}} \mathbf{N}$ are symmetric, and

$$
\mathbf{W}^{\mathrm{t}} \mathbf{W}+\mathbf{X}^{\mathrm{t}} \mathbf{X}+\mathbf{Y}^{\mathrm{t}} \mathbf{Y}+\mathbf{Z}^{\mathrm{t}} \mathbf{Z}=4 w \mathbf{I}_{\mathbf{w}}
$$

Williamson [Wi] constructed Hadamard matrices $H_{n}$ (i.e., $H_{n}^{t} H_{n}=n I_{n}$, $n=4 w$ ), in which the first, second, third and fourth (block) columns are respectively.

$$
\begin{array}{r}
e_{1}=[\mathbf{W},-\mathbf{X},-\mathbf{Y},-\mathbf{Z}], \quad e_{2}=[\mathbf{X}, \mathbf{W}, \mathbf{Z},-\mathbf{Y}], \quad e_{3}=[\mathbf{Y},-\mathbf{Z}, \mathbf{W}, \mathbf{X}] \\
\text { and } e_{4}=[\mathbf{Z}, \mathbf{Y},-\mathbf{X}, \mathbf{W}]
\end{array}
$$

We note that the four column vectors $e_{k}$ are orthogonal, i.e., $e_{i}^{t} \cdot e_{j}=\mathbf{0}$, for $i \neq j$, where $\mathbf{0}$ is the zero matrix and $e_{i}^{t} \cdot e_{i}=\mathbf{W}^{\mathrm{t}} \mathbf{W}+\mathbf{X}^{\mathrm{t}} \mathbf{X}+\mathbf{Y}^{\mathrm{t}} \mathbf{Y}+\mathbf{Z}^{\mathrm{t}} \mathbf{Z}=4 w \mathbf{I}_{\mathbf{w}}$. 
Let $S=\left(s_{k}\right)_{n}=\left(s_{1}, s_{2}, \ldots, s_{n}\right)$ be a sequence of real numbers, then $S(z)=$ $\sum_{k} s_{k} z^{k-1} \quad(1 \leq k \leq n)$ is called the associated polynomial of $S$ and $s(j)=$ $\sum_{k} s_{k} s_{k+j} \quad(1 \leq k \leq n-j)$, the jth nonperiodic auto-correlation function of $S$, where $0 \leq j \leq n-1$, and $s(j)=0$ for $j \geq n$. We note here that $|S|^{2}=S(z) S\left(z^{-1}\right)=s(0)+\sum_{k} s(k)\left(z^{k}+z^{-k}\right) \quad(1 \leq k \leq n-1)$ is the generating function for $s(k)$, where $z \in \mathbf{K}=\{z \in \mathbf{C}:|z|=1\}$, the unit circle, and $\mathbf{C}$ is the complex field. We shall use the same letter to represent a sequence and its associated polynomial.

An $m$-symbol $\delta$-code of length $n$ is a sequence of vectors, $V=\left(v_{1}, v_{2}, \ldots\right.$, $v_{n}$ ), where $v_{k}$ is one of $m$ orthonormal (column) vectors, $i_{1}, i_{2}, \ldots, i_{m}$ or their negatives, such that $v(j)=0$ for $j \neq 0$, where $v(j)=\sum_{k} v_{k}^{t} \cdot v_{k+j} \quad(1 \leq$ $k \leq n-j)$ is the nonperiodic auto-correlation function of $V$.

When $m=4$, i.e., for a 4-symbol $\delta$-code $V$ of length $n$, it is convenient to set $i_{1}=[1,1,0,0], i_{2}=[1,-1,0,0], i_{3}=[0,0,1,1]$ and $i_{4}=[0,0,1,-1]$ as four orthogonal column vectors with normalized length $\sqrt{ } 2$. By letting $v_{k}=$ $\left[q_{k}, r_{k}, s_{k}, t_{k}\right]$, we have

$$
v(j)=q(j)+r(j)+s(j)+t(j)=0 \quad \text { for } j \neq 0,
$$

where $q(j), r(j), s(j)$ and $t(j)$ are respectively the nonperiodic auto-correlation functions of the component sequences of $V$, i.e.,

$$
\begin{array}{r}
Q=\left(q_{1}, \ldots, q_{n}\right), \quad R=\left(r_{1}, \ldots, r_{n}\right), \quad S=\left(s_{1}, \ldots, s_{n}\right) \\
\text { and } T=\left(t_{1}, \ldots, t_{n}\right) .
\end{array}
$$

A $\delta$-code of length $n$ represented by $4 \times n$ matrix $[Q, R ; S, T]$ is called a regular $\delta$-code of length $n$ (abbreviated as $R D(n)$ ), where $Q, R, S$ and $T$ are the four component sequences of a 4-symbol $\delta$-code $V=\left(v_{1}, \ldots, v_{n}\right)$ in (4). We note here that $[Q, R ; 0,0]$ and $[0,0 ; S, T]$ are in symbols $i_{1}, i_{2}$ and $i_{3}, i_{4}$ respectively. Since $q(0)+r(0)+s(0)+t(0)=2 n$, condition (3) is equivalent to

$$
|Q|^{2}+|R|^{2}+|S|^{2}+|T|^{2}=2 n \text { for any } z \text { on the unit circle } \mathbf{K} \text {. }
$$

And we also note that in $V=[Q, R ; S, T]$, either $\left|q_{k}\right|=\left|r_{k}\right|=1$ and $s_{k}=$ $t_{k}=0$, or $q_{k}=r_{k}=0$ and $\left|s_{k}\right|=\left|t_{k}\right|=1$, for each $k$.

A pair of $(1,-1)$-sequences, $\mathbf{F}=\left(f_{1}, \ldots, f_{g}\right)$ and $\mathbf{H}=\left(h_{1}, \ldots, h_{g}\right)$ is called a pair of Golay complementary sequences of length $g$ (abbreviated as $G C S(g)$ ), if their auto-correlation functions satisfy $f(j)+h(j)=0$ for $j \neq 0$ [G], or equivalently $|\mathbf{F}|^{2}+|\mathbf{H}|^{2}=2 g$ for any $z$ on the unit circle $\mathbf{K}$ [Y4]. Golay complementary sequences $G C S(g)$ exist for $g=2^{a} 10^{b} 26^{c}$ (Golay numbers), where $a, b$ and $c$ are nonnegative integers [T1]. For a given pair of Golay complementary sequences $\mathbf{F}$ and $\mathbf{G}$ of length $g,[\mathbf{F}, \mathbf{G} ; 0,0]$ is a regular $\delta$ code $R D(g)$ in symbols $i_{1}$ and $i_{2}$, where $0=0_{g}$ is the sequence of zeros of length $g$. We shall use $g$ for a Golay number.

A square matrix $\mathbf{S}=\left[s_{i j}\right]$ of order $n$ is circulant if $s_{i j}=s_{k+1}$ for $k \equiv j-$ $i(\bmod n)$. We note here that the first, second $, \ldots, n$th rows of $\mathbf{S}$ are respectively, $\quad\left(s_{1}, s_{2}, \ldots, s_{n}\right), \quad\left(s_{n}, s_{1}, \ldots, s_{n-1}\right), \ldots,\left(s_{2}, s_{3}, \ldots, s_{1}\right) . \quad$ Also 
$\mathbf{S}^{\mathrm{t}} \mathbf{S}=\left[p_{i j}\right]$ is symmetric circulant, i.e., $p_{i j}=p_{j i}=s^{*}(h)$, where $h=|i-j|$, and $s^{*}(h)=\sum_{k} s_{k} s_{k+h} \quad(1 \leq k \leq n)$, where the subscript $k+h$ is congruent modulo $n$.

$s^{*}(j)$ is called the $j$ th periodic auto-correlation function of $S=\left(s_{1}, s_{2}, \ldots\right.$, $\left.s_{n}\right)$. We note that $s^{*}(j)=s(j)+s(n-j)=s^{*}(n-j)$ and $s^{*}(0)=s(0)$. Also if $q(j)+r(j)+s(j)+t(j)=0$ for $j \neq 0$ then $q^{*}(j)+r^{*}(j)+s^{*}(j)+t^{*}(j)=0$ for $j \neq 0$.

Let $\mathbf{A}, \mathbf{B}, \mathbf{C}$, and $\mathbf{D}$ be four circulant square matrices of order $m$ with entries \pm 1 satisfying

$$
\mathbf{A}^{\mathbf{t}} \mathbf{A}+\mathbf{B}^{\mathbf{t}} \mathbf{B}+\mathbf{C}^{\mathbf{t}} \mathbf{C}+\mathbf{D}^{\mathbf{t}} \mathbf{D}=4 m \mathbf{I}_{\mathbf{m}} .
$$

Then a Goethals-Seidel (Hadamard) matrix [Go], $G$ of order $4 m$ can be constructed, in which the first, second, third and fourth (block) rows are respectively $(\mathbf{A}, \mathbf{B R}, \mathbf{C R}, \mathbf{D R}),\left(-\mathbf{B R}, \mathbf{A},-\mathbf{D}^{\prime} \mathbf{R}, \mathbf{C}^{\prime} \mathbf{R}\right),\left(-\mathbf{C R}, \mathbf{D}^{\prime} \mathbf{R}, \mathbf{A},-\mathbf{B}^{\prime} \mathbf{R}\right)$ and $\left(-\mathbf{D R},-\mathbf{C}^{\prime} \mathbf{R}, \mathbf{B}^{\prime} \mathbf{R}, \mathbf{A}\right)$, where $\mathbf{R}=\left[r_{i j}\right]$ is the matrix with $r_{i j}=1$ for $i+j=$ $m+1$, and $r_{i j}=0$ otherwise, for $1 \leq i, j \leq m$ and $\mathbf{P}^{\prime}=\left[p_{j i}\right]$ for $\mathbf{P}=\left[p_{i j}\right]$. We note that $\mathbf{P}^{\mathbf{t}} \neq \mathbf{P}^{\prime}$ if $p_{i j}^{t} \neq p_{i j}$, and $G^{t} G=\left(\mathbf{A}^{\mathbf{t}} \mathbf{A}+\mathbf{B}^{\mathbf{t}} \mathbf{B}+\mathbf{C}^{\mathbf{t}} \mathbf{C}+\mathbf{D}^{\mathbf{t}} \mathbf{D}\right) \times \mathbf{I}_{\mathbf{4}}=$ $\left(4 m \mathbf{I}_{\mathrm{m}}\right) \times \mathbf{I}_{\mathbf{4}}=4 m I_{4 m}$.

If Williamson matrices $\mathbf{W}, \mathbf{X}, \mathbf{Y}$ and $\mathbf{Z}$ satisfying (1) and a 4-symbol $\delta$-code $R D(n)$ of (4) exist, then we can construct a Goethals-Seidel (Hadamard) matrix $G$ of order $4 m(m=n w)$ with entries from $\Omega=\{ \pm \mathbf{W}, \pm \mathbf{X}, \pm \mathbf{Y}, \pm \mathbf{Z}\}$, by finding matrices $\mathbf{A}, \mathbf{B}, \mathbf{C}$ and $\mathbf{D}$ satisfying (5), as follows. The first rows of circulant matrices $\mathbf{A}, \mathbf{B}, \mathbf{C}$ and $\mathbf{D}$ of order $m=n w$ are respectively the corresponding component sequences $A, B, C$ and $D$ obtained by replacing the four orthogonal column vectors $i_{k}, 1 \leq k \leq 4$, with the four orthogonal column vectors $e_{k}$ of (2) in the $\delta$-code $R D(n)$ of (4). Let $A=\left(a_{1}, \ldots, a_{n}\right)$, $B=\left(b_{1}, \ldots, b_{n}\right), C=\left(c_{1}, \ldots, c_{n}\right)$ and $D=\left(d_{1}, \ldots, d_{n}\right)$, where $a_{k}, b_{k}, c_{k}$ and $d_{k}$ are from $\Omega$. Then the following conditions corresponding to (3) hold:

$$
\begin{array}{r}
a(j)+b(j)+c(j)+d(j)=0 \quad \text { for } j \neq 0, \quad \text { where } s(j)=\sum_{k} s_{k}^{t} \cdot s_{k+j} \\
(1 \leq k \leq n-j), s_{k} \in \Omega,
\end{array}
$$

and

$$
a(0)+b(0)+c(0)+d(0)=n\left(\mathbf{W}^{\mathbf{t}} \mathbf{W}+\mathbf{X}^{\mathbf{t}} \mathbf{X}+\mathbf{Y}^{\mathbf{t}} \mathbf{Y}+\mathbf{Z}^{\mathbf{t}} \mathbf{Z}\right)=4 n w \mathbf{I}_{\mathbf{w}} .
$$

Also for any circulant matrix $\mathbf{S}$ of order $n$ having the first row $\left(s_{1}, \ldots, s_{n}\right)$ with entries $s_{k} \in \Omega$, we have $\mathbf{S}^{t} \mathbf{S}=\left[s^{*}(h)\right]$, where $h=|i-j|, s^{*}(h)=$ $s(h)+s(n-h)$ and $s^{*}(0)=s(0)$, consequently

$$
\begin{aligned}
\mathbf{A}^{\mathbf{t}} \mathbf{A}+\mathbf{B}^{\mathbf{t}} \mathbf{B}+\mathbf{C}^{\mathbf{t}} \mathbf{C}+\mathbf{D}^{\mathbf{t}} \mathbf{D} & =\left[a^{*}(h)+b^{*}(h)+c^{*}(h)+d^{*}(h)\right] \\
& =\left(4 n w \mathbf{I}_{\mathbf{w}}\right) \times \mathbf{I}_{\mathbf{n}}=4 n w \mathbf{I}_{\mathbf{n} \mathbf{w}} .
\end{aligned}
$$


A quadruple of $(1,-1)$-sequences $(A, B ; C, D)$ respectively with lengths $m+p$ and $m$ pairs, where

$$
\begin{array}{r}
A=\left(a_{1}, \ldots, a_{m+p}\right), \quad B=\left(b_{1}, \ldots, b_{m+p}\right), \quad C=\left(c_{1}, \ldots, c_{m}\right) \\
\text { and } \quad D=\left(d_{1}, \ldots, d_{m}\right), \quad p \geq 0,
\end{array}
$$

is called a set of (Turyn) base sequences for length $t=2 m+p$ (abbreviated as $B S(t))$, if they have zero auto-correlation sum i.e., $a(j)+b(j)+c(j)+d(j)=0$ for $j \neq 0$, or equivalently if $|A|^{2}+|B|^{2}+|C|^{2}+|D|^{2}=2 t$ for any $z$ on $\mathbf{K}$. Sets of base sequences $B S(t)$ are known (published) for $t \in\{3, \ldots, 47,51,53,59$, and $2 g+1: g=2^{a} 10^{b} 26^{c}$ (Golay numbers), $a, b, c$ nonnegative integers $(t=2 m+1)$ with lengths $m+1, m$ pairs and $t=g+g^{\prime}$, where $g$ and $g^{\prime}$ are any Golay numbers (e.g., we may take $g^{\prime}=1$ ). [See below for the cases $t=37,39,43$ and 45.]

From the above $B S(t):(A, B ; C, D), t=2 m+p$, we can obtain the following regular $\delta$-codes $R D(t):[Q, R ; S, T]$, trivially.

$$
Q=(A, 0), \quad R=(B, 0), \quad S=(\mathbf{0}, C) \text { and } T=(\mathbf{0}, D),
$$

where $0=0_{m}=$ the sequence of zeros of length $m$, and $\mathbf{0}=0_{m+p}$; and when $p=1$ or 0 , i.e., with lengths $m+1$ and $m$ pairs or with $m$ and $m$ pairs.

$$
Q=(A / 0), \quad R=(B / 0), \quad S=(\mathbf{0} / C) \text { and } T=(\mathbf{0} / D),
$$

where $(X / Y)$ means the interleaved sequence $\left(x_{1}, y_{1}, \ldots, x_{k}, y_{k}, \cdots\right)$ for $X=\left(x_{1}, \ldots, x_{k}, \cdots\right)$ and $Y=\left(y_{1}, \ldots, y_{k}, \ldots\right)$. The $\delta$-codes (6) and (7) are called Turyn $\delta$-codes of length $t$.

The following Lagrange identity for polynomials is the key for composition of 4-symbol $\delta$-codes and other related codes [Y1,Y2].

Theorem $\mathbf{L}$ (Lagrange identity for polynomials). Let $a, b, c, d, e, f, g$ and $h$ be polynomials in $z$ with real coefficients. Also let $p^{\prime}=p\left(z^{-1}\right)$ for $p=p(z)$ and

$$
\begin{array}{ll}
\mathbf{q}=-b^{\prime} e+a f^{\prime}+c g+d h, & \mathbf{s}=-d^{\prime} e-c f+a g^{\prime}-b h, \\
\mathbf{r}=a^{\prime} e+b f^{\prime}+d g^{\prime}-c h^{\prime}, & \mathbf{t}=c^{\prime} e-d f+b g+a h^{\prime} .
\end{array}
$$

Then $|\mathbf{q}|^{2}+|\mathbf{r}|^{2}+|\mathbf{s}|^{2}+|\mathbf{t}|^{2}=\left(|a|^{2}+|b|^{2}+|c|^{2}+|d|^{2}\right)\left(|e|^{2}+|f|^{2}+|g|^{2}+|h|^{2}\right)$ for any $z$ on $\mathbf{K}$.

Let $P^{*}=\left(p_{1}^{*}, \ldots, p_{n}^{*}\right)$, where $p_{k}^{*}=p_{n+1-k}$, be the reverse of a sequence $P=\left(p_{1}, \ldots, p_{n}\right)$. We note that $P^{\prime}=P\left(z^{-1}\right)=\sum p_{k} z^{1-k}=z^{1-n}\left(\sum p_{k}^{*} z^{k-1}\right)=$ $z^{1-n} P^{*}(z)$; consequently $\left|P^{*}\right|^{2}=|P|^{2}$.

A $(0, \pm 1)$-sequence of length $n, S=\left(s_{k}\right)=\left(s_{1}, \ldots, s_{n}\right)$ is symmetric if $s_{k}^{*}=s_{k}$ for each $k$, (i.e., $S^{*}=S$ ) ; it is skew if $s_{k}^{*}=-s_{k}$ for each $k$, (i.e., $S^{*}=-S$ ); and it is said to be quasi-symmetric if $s_{k}^{*}=e_{k} s_{k}$ for each $k$, where $e_{k}=1$ or -1 , i.e., zeros appear symmetrically in $S$. Consequenlty symmetric or skew sequences are quasi-symmetric. Two $(0, \pm 1)$-sequences of 
length $n, G=\left(g_{k}\right)$ and $H=\left(h_{k}\right)$ are said to be supplementary if $G+H=$ $\left(g_{k}+h_{k}\right)$ is a $(1,-1)$-sequence; i.e., $\left|g_{k}\right|=1$ and $h_{k}=0$, or $g_{k}=0$ and $\left|h_{k}\right|=1$, for each $k$. We note here that $Q, R$ and $S, T$ are supplementary in a regular $\delta$-code: $[Q, R ; S, T]$; i.e., $G+H$ is a $(1,-1)$-sequence for $G=Q$ or $R$, and $H=S$ or $T$.

Definition 1. A triple $(F ; G, H)$ of sequences is said to be a set of normal sequences for length $n$ (abbreviated as $N S(n)$ ) if the following two conditions are satisfied.

(i) $\quad F=\left(f_{k}\right)$ is a $(1,-1)$-sequence of length $n ; G=\left(g_{k}\right)$ and $H=$ $\left(h_{k}\right)$ are quasi-symmetric supplementary $(0, \pm 1)$-sequences of length $n$; i.e., $G+H=\left(g_{k}+h_{k}\right)$ is a $(1,-1)$-sequence and zeros appear symmetrically in $G$ and $H$.

(ii) $f(j)+g(j)+h(j)=0$ for $j \neq 0$, i.e., they have zero auto-correlation sum.

Condition (ii) is also equivalent to

(iii) $|F|^{2}+|G|^{2}+|H|^{2}=2 n$ for any $z$ on $\mathbf{K}$.

It is known that if $J=L+M$ is a sequence of real numbers with symmetric $L$ and skew $M$, then $|J|^{2}=|L|^{2}+|M|^{2}$ for any $z$ on $\mathbf{K}$. From a given pair of Golay sequences $G C S(g):(\mathbf{F}, \mathbf{H})$, we can obtain trivially two sets of normal sequences, $\left(\mathbf{F} ; \mathbf{H}, 0_{g}\right)$ and $(\mathbf{F} ; L, M)$, where $L$ and $M$ are respectively the symmetric and skew parts of $\mathbf{H}$. Therefore, a set of normal sequences can be regarded as a generalization of a pair of Golay complementary sequences. We can also obtain sets of normal sequences $N S(2 m+1):\left(A / C ; B / 0_{m}, 0_{m+1} / D\right)$ from Turyn base sequences $T B S(2 m+1):(A, B ; C, D)$ with lengths $m+1$ and $m$ pairs, for even $m$ with symmetric $A$ and skew $C$, and for odd $m$ with skew $A$ and symmetric $C$. It is known that $T B S(2 m+1)$ with such properties exists for $m \leq 7, m=14$ [T1] and $m=12$ [GS]. For example, from $T B S(3):(A, B ; C, D)=(1-, 11 ; 1,1)$, where - stands for -1 , we obtain $N S(3)$ : $F=A / C=11-; G=B / 0=101, H=0_{2} / D=010$, where $m=1$ is odd, $A=1-$ is skew and $C=1$ is symmetric. Also from $T B S(5):(111,11-; 1-, 1-)$, we obtain $N S(5): F=A / C=111-1$; $G=B / 0_{2}=1010-, H=0_{3} / D=010-0$, where $m=2$ is even, $A=111$ is symmetric and $C=1-$ is skew. Also from $G C S(4):(\mathbf{F}, \mathbf{H})=(111-, 11-1)$, we get $(\mathbf{F} ; L, M)=(111-; 1001,01-0)$, where $L=1001$ and $M=01-0$ are respectively symmetric and skew parts of $\mathbf{H}$.

The following are examples of $N S(n):(F ; G, H)$, which are unobtainable from $T B S$ and $G C S$.

$n=7: 1-111--; 11010-1,0010100$ and $11--1-1 ; 101110-$, 0100010 , as $F ; G, H$.

$$
\begin{aligned}
& n=9: 1-11111--; 11-0101-1,00010-000 . \\
& n=11: 1111-1--11-; 1100-0100-1,00110101-00 . \\
& n=12: 111---1--1--; 11000000-11,00011-1-1000 . \\
& n=13: 111----1---1-; 111-0000011-1,00001-11-0000 .
\end{aligned}
$$


Definition 2. A quadruple $(E, F ; G, H)$ of $(0, \pm 1)$-sequences is said to be a set of near normal sequences for length $n=4 m+1$ (abbreviated as $N N(n)$ ) if the following conditions are satisfied.

(i) $E=(X / 0,1), F=(Y / 0)$, where $X$ and $Y$ are $(1,-1)$-sequences of length $m$ and $0=0_{m-1}$, the sequence of zeros of length $m-1$, i.e., $E$ and $F$ are respectively of lengths $2 m$ and $2 m-1 ; G$ and $H$ are quasisymmetric supplementary $(0, \pm 1)$-sequences of length $2 m$, i.e., $G+H$ is a $(1,-1)$-sequence of length $2 m$ and zeros appear symmetrically in $G$ and $H$.

(ii) $e(j)+f(j)+g(j)+h(j)=0$ for $j \neq 0$, i.e., they have zero autocorrelation sum. Condition (ii) is also equivalent to

(iii) $|E|^{2}+|F|^{2}+|G|^{2}+|H|^{2}=4 m+1$ for any $z$ on $\mathbf{K}$.

The following are examples of sets of near normal sequences $N N(n):(E, F$; $G, H)$. The cases for $n \geq 21$ were found by a computer search. (All $N N(n)$ for $n \leq 37$ have been found and classified in [Y5].

$$
\begin{aligned}
& n=5: 11,1 ; 1-, 00, \text { as } E, F ; G, H, \text { where }- \text { stands for }-1 . \\
& n=9:-011,101 ; 1001,01-0 . \\
& n=13: 10-011,10101 ; 11-1--, 0_{6} . \\
& n=17:-0-01011,10-010-; 111-1-11,0_{8} . \\
& n=21: 1010-01011,101010-0-; 10101-010-, 010-0010-0 . \\
& n=25: 10101010-011,1010-0-0-01 ; 110_{8}--, 001--11-1-00 . \\
& n=29: 10101010-010-1,1010-0101010-; 1-0_{10} 1-, 0011-1111- \\
& -00 .
\end{aligned}
$$$$
n=33:-0-010101010-011,101010-010-0101 ; 1001--01-0---001,
$$
$01-0001001000-10$.

$n=37:-010101010101010-1,1010-0-01010-010-; 1101---$ $001--110-1,0010_{5} 1-0,-00$.

$n=41: 1010-0-010101010-011,-0101010101010-010-; 110-10-$ $-1-1--10110--, 0010010_{8}-00-00$.

$n=45: 10-0-010-010-010101011,-01010-01010-0-010101$; $0010_{5} 10010_{5}-001,0-101-1--01101111-01-0$.

We note that we can obtain base sequences $B S(2 n+1):(A, B ; C, D)$ with lengths $n+1, n$ pairs, from a set of normal sequences $N S(n):(F ; G, H)$ as follows: $A=(F, 1), B=(F,-1) ; C=G+H, D=G-H$. And $B S(n)$ from a set of near normal sequences $N N(n):(E, F ; G, H)$, where $E=(X / 0,1)$ and $F=(Y / 0)$, as follows: $A=F / E=(Y / X, 1), B=(-F) / E=((-Y) / X, 1)$ $C=G+H, D=G-H$. For example, from the above $N S(12)$, we obtain the following new construction for $B S(25): A=111---1--1--1$, $B=111---1--1---; C=11111-1-1-11, D=111--1-1--11$. Also from the above $N N(n)$, we obtain the following new construction for $B S(n), n=29,37$ and 45 . 


$$
\begin{aligned}
& n=29: 1111-1111-11--1,-1-111-1---11-1 ; 1-11-1111---1-, \\
& 1---1----1111-\text { as }(A, B ; C, D) . \\
& n=37: 1-11-1-11111-111--1,---11111-1-111-11-1 ; \\
& 1111---1-1--11,--1,11-1-----11--111-1 . \\
& \quad n=45:-11-1--11-11---11111111,11----11---11-11-1- \\
& 1-11 ;--111-1--11111111--1-1,-1-1-1-111--1----1--11 .
\end{aligned}
$$

(For $n=37$ also see [K].) The only unpublished among $B S(p)$ for all primes $p \leq 59$ is $p=43$, so we give these as follows. $p=43: 11-1-1-------1-$ $-11-1--, 11----1-1-11---1-111-1 ; 1-11-111111---11--111$, $11-1-1111--1--11----1$. We also give another previously unpublished case, $B S(39)$ as follows: $11-1-1-111--1--1-1--, 11----1---$ $11-11111-1 ; 1-11-11--111-1-1111,11-----111--1-----1$.

\section{General Results}

For given $B S(t):(A, B ; C, D)$ with lengths $m+p$ and $m$ pairs, $t=2 m+p$, and $N S(n):(F ; G, H)$ with $F=\left(f_{k}\right)$ and $G+H=\left(g_{k}+h_{k}\right)=\left(j_{k}\right)$, let

$\left(i^{*}\right)$ when $j_{k}=g_{k}: \alpha j_{k}=A g_{k}^{*}, \beta j_{k}=B g_{k}, \gamma j_{k}=C g_{k}, \delta j_{k}=D g_{k}^{*}$;

(ii*) when $j_{k}=h_{k}: \alpha j_{k}=-B h_{k}, \beta j_{k}=A h_{k}^{*}, \gamma j_{k}=D h_{k}, \delta j_{k}=-C h_{k}^{*}$.

We also define five $2 \times t$ matrices as follows.

$$
\begin{aligned}
\sigma_{k} & =\left(\begin{array}{ll}
A f_{k}^{*}, & \gamma j_{k} \\
B f_{k}^{*}, & \delta j_{k}
\end{array}\right), \quad \tau_{k}=\left(\begin{array}{l}
\alpha j_{k},-C f_{k}^{*} \\
\beta j_{k},-D f_{k}^{*}
\end{array}\right), \\
0 & =\left(\begin{array}{l}
\mathbf{0}, \underline{0} \\
\mathbf{0}, \underline{0}
\end{array}\right), \quad \varepsilon_{1}=\left(\begin{array}{rr}
-B^{*}, \underline{0} \\
A^{*}, \underline{0}
\end{array}\right), \quad \varepsilon_{2}=\left(\begin{array}{rr}
\mathbf{0}, & D^{*} \\
\mathbf{0}, & C^{*}
\end{array}\right),
\end{aligned}
$$

where $\underline{0}=0_{m}$ and $\mathbf{0}=0_{m+p}$.

Theorem 1. Let $(A, B ; C, D)$ be base sequences $B S(t)$ with lengths $m+p$ and $m$ pairs, $t=2 m+p$, and $(F ; G, H)$ be normal sequences $N S(n)$. Then the following $4 \times(s t)$ matrix $[Q, R ; S, T]$ is a regular $\delta$-code $R D(s t), s=2 n+1$, where $[Q, R]$ and $[S, T]$ are column vectors, i.e., $2 \times(s t)$ matrices.

$$
\begin{gathered}
{[Q, R]=\left[\left(q_{k}\right),\left(r_{k}\right)\right]=\left(\sigma_{1}, 0, \sigma_{2}, 0, \ldots, \sigma_{n}, 0, \varepsilon_{1}\right),} \\
{[S, T]=\left[\left(s_{k}\right),\left(t_{k}\right)\right]=\left(0, \tau_{1}, 0, \tau_{2}, \ldots, 0, \tau_{n}, \varepsilon_{2}\right),}
\end{gathered}
$$

where $\sigma_{k}, \tau_{k}, \varepsilon_{k}$ and 0 are defined in $(*)$.

Proof. In (I), each column vector $\left[q_{k}, r_{k}, s_{k}, t_{k}\right]$ is obviously one of orthogonal vectors $i_{h}$ or their negatives, $1 \leq h \leq 4$. In (L), let $a=A(z), b=B(z)$, $c=C(z) z^{M}$, and $d=D(z) z^{M}$. Also let $f=F\left(z^{2 t}\right) z^{-x}, g=G\left(z^{2 t}\right) z^{-x}$, $h=H\left(z^{2 t}\right) z^{-x}$ and $e=z^{y}$, where $t=2 m+p, M=m+p, x=(n-1) t$ and $y=(n+1) t+M-1$. Then we have $Q=\mathbf{q} z^{x}, R=\mathbf{r} z^{x}, S=\mathbf{s} z^{x+t}$ and $T=\mathbf{t} z^{x+t}$.

Consequently we obtain from Theorem $L,|Q|^{2}+|R|^{2}+|S|^{2}+|T|^{2}=|\mathbf{q}|^{2}+$ $|\mathbf{r}|^{2}+|\mathbf{s}|^{2}+|\mathbf{t}|^{2}=\left(|A|^{2}+|B|^{2}+|C|^{2}+|D|^{2}\right)\left(1^{2}+|F|^{2}+|G|^{2}+|H|^{2}\right)=2 s t$ for any $z$ on $\mathbf{K}$. Thus the theorem is proved. 
We note here that $p=p(z)=P\left(z^{2 t}\right) z^{-x}$ is symmetrized, i.e., $p^{\prime}=p\left(z^{-1}\right)=$ $P^{*}\left(z^{2 t}\right) z^{-x}$ for $p=f, g$ and $h$. And in $\mathbf{q}, \mathbf{r}, \mathbf{s}$ and $\mathbf{t}$, the quasi-symmetry of sequence $P=\left(p_{k}\right)$ is required for $P=F, G$ and $H$, since $p_{k}$ and $p_{k}^{*}$ of the reverse $P^{*}$ determine the $k$ th block of length $2 t$ and align its nonzero and zero parts. Similarly $G$ and $H$ must be supplementary. Therefore quasi-symmetry and supplementary are essential for the construction of a $\delta$-code in Theorem 1 .

A set of four $(1,-1)$-sequences $(U, W, X, Y)$ of length $m$ is said to be complementary, if $u(j)+w(j)+x(j)+y(j)=0$ for $j \neq 0$, or equivalently, if $|U|^{2}+|W|^{2}+|X|^{2}+|Y|^{2}=4 m$ for any $z$ on $\mathbf{K}$. The sequences $U, W, X$ and $Y$ can be regarded as respectively the first rows of circulant matrices $A, B, C$ and $D$ of order $m$ satisfying condition (5), i.e., $A^{t} A+B^{t} B+C^{t} C+D^{t} D=4 m I_{m}$, thus a Goethals-Seidel (Hadamard) matrix of order $4 m$ can be constructed. Also we note here that the above $(U, W ; X, Y)$ can be regarded as $B S(2 m)$ with lengths $m$ and $m$ pairs.

Theorem 2. Let $B S(t)$ and $N S(n)$ be given as in Theorem 1. Then the following $(Q, R, S, T)$ is a set of four complementary sequences of length $n t$ (i.e., BS(2nt) with lengths $n t$ and $n t$ pairs).

$$
[Q, R]=\left(\sigma_{1}, \sigma_{2}, \ldots, \sigma_{n}\right)
$$$$
[S, T]=\left(\tau_{1}, \tau_{2}, \ldots, \tau_{n}\right)
$$

where $\sigma_{k}$ and $\tau_{k}$ are defined in $(*)$.

The proof of Theorem 2 is similar to that of Theorem 1, therefore we omit it. We note here that $z^{t}$ should be used instead of $z^{2 t}$ in $F, G$ and $H$; and $e=0$, consequently there are no $A^{*}, B^{*}, C^{*}$ and $D^{*}$ in (II). Also there are no shifting for $S$ and $T$, i.e., $S=\mathbf{s} z^{x}$ and $T=\mathbf{t} z^{x}, x=(n-1) t / 2$.

Let the four components of a regular $\delta$-code $R D(u):[\mathbf{A}, \mathbf{B} ; \mathbf{C}, \mathbf{D}]$ be

$$
\left(\mathrm{II}^{*}\right) \quad \mathbf{A}=(\underline{A}, \mathbf{0}), \quad \mathbf{B}=(\underline{B}, \mathbf{0}) ; \quad \mathbf{C}=(\mathbf{0}, \underline{C}) \quad \text { and } \quad \mathbf{D}=(\mathbf{0}, \underline{D}) \text {, }
$$

where $\underline{P}$ is the part of $\mathbf{P}=\left(p_{k}\right)$ in which $p_{k} \neq 0$ and $\mathbf{O}$ is that in which $p_{k}=0$, for $\mathbf{P} \in\{\mathbf{A}, \mathbf{B}, \mathbf{C}, \mathbf{D}\}$. Thus $\mathbf{X}+\mathbf{Y}=(\underline{X}, \underline{Y})$ for $\mathbf{X} \in\{ \pm \mathbf{A}, \pm \mathbf{B}\}$ and $\mathbf{Y} \in\{ \pm \mathbf{C}, \pm \mathbf{D}\}$, since $\mathbf{X}$ and $\mathbf{Y}$ are supplementary.

For example, in $R D(3 t): \mathbf{A}=\left(A, C ; 0,0 ;-B^{*}, 0\right), \mathbf{B}=\left(B, D ; 0,0 ; A^{*}, 0\right)$; $\mathbf{C}=\left(0,0 ; A,-C ; 0,-D^{*}\right)$ and $\mathbf{D}=\left(0,0 ; B,-D ; 0, C^{*}\right)$, we have $(\mathbf{A}, \mathbf{C})=$ $\mathbf{A}+\mathbf{C}=\left(A, C ; A,-C ;-B^{*},-D^{*}\right)=(\underline{A}, \underline{C})$, etc.

By observing that in Theorem 2 our argument is still valid if we replace $B S(t):(A, B ; C, D)$ by $R D(u)$ of $\left(\right.$ II $\left.^{*}\right)$, i.e., by replacing $P$ of $B S$ with $\underline{P}$ of $R D$ for $\mathbf{P} \in\{\mathbf{A}, \mathbf{B}, \mathbf{C}, \mathbf{D}\}$ and $t$ with $u$.

Consequently we have the following.

Theorem $2^{*}$. Let $[\mathbf{A}, \mathbf{B} ; \mathbf{C}, \mathbf{D}]$ be a regular $\delta$-code $R D(u)$ of $\left(\mathrm{II}^{*}\right)$, then $(Q, R, S, T)$ of (II) is a set of four complementary sequences of length nu (or equivalently $B S(2 n u))$.

We note here that, from condition (iii) of Definition 1, a set of normal sequences $N S(2 m)$ exists only if $4 m$ is the sum of three squares of even integers, consequently $m$ must be the sum of three squares of integers. Therefore 
$N S(2 m)$ does not exist for $m \equiv 7(\bmod 8)$. It is known that $N S(6)$ does not exist. Although $R D(n t)$ cannot be composed by Theorem 1 for $n=2 k+1$, $k=6$ or 14 (since $N S(k)$ does not exist for these cases), $R D(n t)$ can be constructed for $n=4 m+1$, which includes $13,29, \ldots$, by the following theorem with a set of near normal sequences $N N(n)$.

Theorem 3. Let $(A, B ; C, D)$ be a set of base sequences $B S(t)$ with lengths $s+p$ and $s$ pairs, and $(E, F ; G, H)$ be a set of near normal sequences $N N(n)$ of Definition 2, where $n=4 m+1$ and $t=2 s+p$. Then the following $4 \times(n t)$ matrix $[Q, R ; S, T]$ is a regular $\delta$-code $R D(n t)$.

$$
\begin{aligned}
& {[Q, R]=\left(\lambda_{1}, \mu_{1}, \ldots, \lambda_{m}, \mu_{m}, \varepsilon_{2}, 0,0, \ldots, 0,0\right),} \\
& {[S, T]=\left(0,0, \ldots, 0,0, \varepsilon_{3}, \nu_{m}, \pi_{m}, \ldots, \nu_{1}, \pi_{1}\right),}
\end{aligned}
$$

where

$$
\begin{array}{rlrl}
\lambda_{k} & =\left(\begin{array}{cc}
\alpha j_{2 k-1}, & -C y_{k} \\
\beta j_{2 k-1}, & -D y_{k}
\end{array}\right), & \mu_{k} & =\left(\begin{array}{cc}
\alpha j_{2 k}, & -D^{*} x_{k} \\
\beta j_{2 k}, & C^{*} x_{k}
\end{array}\right), \\
\nu_{k} & =\left(\begin{array}{cc}
-B x_{k}, & \gamma^{*} j_{2 k} \\
A x_{k}, & \delta^{*} j_{2 k}
\end{array}\right), & \pi_{k}=\left(\begin{array}{cc}
A^{*} y_{k}^{*}, & \gamma^{*} j_{2 k-1} \\
B^{*} y_{k}^{*}, & \delta^{*} j_{2 k-1}
\end{array}\right), \\
\varepsilon_{2}=\left(\begin{array}{rr}
\mathbf{0},-D^{*} \\
\mathbf{0}, & C^{*}
\end{array}\right) \text { and } & \varepsilon_{3}=\left(\begin{array}{c}
-B, \underline{0} \\
A, \underline{0}
\end{array}\right) .
\end{array}
$$

Also $\xi j_{i}$, for $\xi=\alpha, \beta, \gamma$ and $\delta$, are defined as in $\left(\mathrm{i}^{*}\right)$ and $\left(\mathrm{ii}^{*}\right)$, where $\xi^{*} j_{i}=\left(\xi j_{i}\right)^{*}$ for $1 \leq i \leq 2 m$, and $y_{k}^{*}=y_{m+1-k}$ for $1 \leq k \leq m$; we note that 0 and $\varepsilon_{2}$ are similar to those defined in $(*)$, i.e., $\mathbf{0}=0_{s+p}$ and $\underline{0}=0_{s}$.

Proof. Obviously each column vector in (III) is one of orthogonal vectors or their negatives $\pm i_{k}$. In $(\mathrm{L})$, let $p=P(z)$ for $p=a, b, c$ and $d$. Also let $f=F\left(z^{t}\right) z^{-u}, e=E\left(z^{t}\right) z^{-u}, g=G\left(z^{t}\right) z^{-x}$ and $h=H\left(z^{t}\right) z^{-x}$, where $t=2 s+p, u=(m-1) t-(p / 2), w=(2 m-5) t / 2+1$, and $x=(2 m-1) t / 2$. Then $Q=\mathbf{s} z^{x}, R=\mathbf{t} z^{x}, S=\mathbf{q}^{\prime} z^{y}$ and $T=\mathbf{r}^{\prime} z^{y}$, where $y=3(2 m+1) t / 2-1$. Consequently, $|Q|^{2}+|R|^{2}+|S|^{2}+|T|^{2}=|\mathbf{s}|^{2}+|\mathbf{t}|^{2}+|\mathbf{q}|^{2}+|\mathbf{r}|^{2}=\left(|A|^{2}+|B|^{2}+\right.$ $\left.|C|^{2}+|D|^{2}\right)\left(|E|^{2}+|F|^{2}+|G|^{2}+|H|^{2}\right)=2 n t$ for any $z$ on $\mathbf{K}$.

We note here that because of the quasi-symmetry of sequences $F, G$ and $H$, in $\mathbf{q}, \mathbf{r}, \mathbf{s}$ and $\mathbf{t}$, the first $2 m$ nonzero blocks of length $t$ are aligned and in the $(2 m+1)$ th (last) block, the nonzero (or zero) part in $\mathbf{q}, \mathbf{r}$ and the zero (or nonzero) part in $\mathbf{s , t}$ are of the same length, thus $\mathbf{q}^{\prime}, \mathbf{r}^{\prime}$ and $\mathbf{s}, \mathbf{t}$ can be fitted to from a $\delta$-code.

From given two sets of base sequences with lengths $m+1, m$ pairs and $n+1, n$ pairs, we can also construct four complementary $(1,-1)$-sequences of length $(2 m+1)(2 n+1)$ as follows.

Theorem 4. Let $(A, B ; C, D)$ and $(F, G ; H, E)$ be two sets of base sequences respectively with lengths $m+1, m$ pairs and $n+1, n$ pairs. Then the following $(Q, R, S, T)$ are four complementary $(1,-1)$-sequences of length $(2 m+1)(2 n+1)$. 


$$
\begin{gathered}
{[Q, R]=\left(\alpha_{1}, \beta_{1}, \alpha_{2}, \beta_{2}, \ldots, \alpha_{n}, \beta_{n}, \alpha_{n+1}\right),} \\
{[S, T]=\left(\gamma_{1}, \delta_{1}, \gamma_{2}, \delta_{2}, \ldots, \gamma_{n}, \delta_{n}, \gamma_{n+1}\right),}
\end{gathered}
$$

where

$$
\begin{aligned}
\alpha_{k} & =\left(\begin{array}{c}
A f_{k}^{*} / C g_{k} \\
B f_{k}^{*} / D g_{k}^{*}
\end{array}\right), \quad \beta_{k}=\left(\begin{array}{c}
-B^{*} e_{k} / D h_{k} \\
A e_{k} /-C h_{k}^{*}
\end{array}\right), \\
\gamma_{k} & =\left(\begin{array}{c}
A g_{k}^{*} /-C f_{k} \\
B g_{k} /-D f_{k}
\end{array}\right) \quad \text { and } \delta_{k}=\left(\begin{array}{c}
-B h_{k} /-D^{*} e_{k} \\
A h_{k}^{*} / C^{*} e_{k}
\end{array}\right) .
\end{aligned}
$$

Proof. Obviously $(Q, R, S, T)$ are four $(1,-1)$-sequences of length $(2 m+$ 1) $(2 n+1)$. By letting $a=A\left(z^{2}\right), b=B\left(z^{2}\right), c=z C\left(z^{2}\right)$ and $d=z D\left(z^{2}\right)$, also $f=z^{-n M} F\left(z^{2 M}\right), g=z^{-n M} G\left(z^{2 M}\right), h=z^{x} H\left(z^{2 M}\right)$ and $e=z^{y} E\left(z^{2 M}\right)$, where $M=2 m+1, x=(1-n) M$ and $y=2 m+(1-n) M$, in (L), and by observing that $Q=\mathbf{q} w, R=\mathbf{r} w, S=\mathbf{s} w$ and $T=\mathbf{t} w$, where $w=z^{n M}$, we obtain

$$
\begin{aligned}
|Q|^{2}+|R|^{2}+|S|^{2}+|T|^{2} & =|\mathbf{s}|^{2}+|\mathbf{t}|^{2}+|\mathbf{q}|^{2}+|\mathbf{r}|^{2} \\
& =\left(|A|^{2}+|B q|^{2}+|C|^{2}+|D|^{2}\right)\left(|E|^{2}+|F|^{2}+|G|^{2}+|H|^{2}\right) \\
& =4(2 m+1)(2 n+1) \quad \text { for any } z \text { on } \mathbf{K} .
\end{aligned}
$$

$B S(t)$ with $m+1$ and $m$ pairs $(t=2 m+1)$ exist for $t \leq 59$ (except $49,57)$ and $t=2 g+1$ [T1,T2].

From given sets of base sequences $B S(t):(A, B ; C, D)$ and normal sequences $N S(m)$ (or near normal sequences $N N(n)$ ), we can construct a regular $\delta$-code $R D((2 m+1) t)$ by Theorem 1 (or $R D(n t)$ by Theorem 3 ). I.e., we can construct $R D(u t)$, where $u \in \mathbf{U}=$ (the set of $2 m+1$ and $n$ such that $N S(m)$ or $N N(n)$ exists $)=\{n: n \leq 33, n=37,41,45,51,53,59,65,81, \ldots$, and $n=2 g+1\}$.

Thus, we can construct $B S(2 m u t)$ by Theorem $2^{*}$ with $N S(m)$ and $R D(u t)$, where $u \in \mathbf{U}$ and $m \in \mathbf{M}=$ (the set of all $m$ such that $N S(m)$ exists $)=\{n$ :odd $n \leq 15, n=25,29 ; n=12$ or $n=g\}$. We note also that from given $R D(u t)$ and Golay sequences $G C S(g)$, four complementary $(1,-1)$ sequences of length $(2 g+1) u t$, equivalently $B S(2(2 g+1) u t)$ can be constructed [Y2].

Consequently from the above, we can obtain $B S\left(t_{1}\right)$, with $t_{1}=2 s_{1} t, s_{1} \in \mathbf{S}$, where

$$
\mathbf{S}=\{m u: m \in \mathbf{M} \text { or } m=2 g+1, u \in \mathbf{U}\},
$$

which contains all positive integers $\leq 100$ (except 43, 47 and primes $\geq 61$ ).

By repeating the above process, we obtain $B S\left(t_{2}\right)$, where $t_{2}=2 s_{2} t_{1}=$ $2^{2} s_{1} s_{2} t, s_{2} \in \mathbf{S}$.

After repeating the process $n$ times, we have the following: 
Theorem 5. Four-symbol $\delta$-codes of length $r$ exist for $r=2^{n} t \prod_{j} s_{j}(1 \leq j \leq n)$, where $s_{j} \in \mathbf{S}, t \in T=\left\{k: k \leq 59\right.$ (except 49,57) or $\left.k=g+g^{\prime}\right\}, g$ and $g^{\prime}$ are Golay numbers; and $\mathbf{S}$ is defined in (IV).

We note that instead of the above, $s_{1}$ and $t$ may belong to $T_{0}=\{h: h \leq 59$ (except 49,57) or $h=2 g+1\}$, since $B S\left(2 s_{1} t\right)$ can be constructed by Theorem 4; also $k=g+g^{\prime}$ includes odd $g+1$, when $g^{\prime}=1$.

\section{EXAMPLES AND REMARK}

From given $B S(t):(A, B ; C, D)$ (or $R D(u))$, and $N S(3):(-11 ; 101,010)$, we obtain the following $[Q, R ; S, T]$ as $R D(7 t)$ from Theorem 1 and as four complementary sequences of length $3 t$ (or $3 u$ ) from Theorem 2 (or Theorem $2^{*}$ ) respectively.

$$
\begin{aligned}
& Q=\left(A, C ; 0,0 ; A, D ; 0,0 ;-A, C ; 0,0 ;-B^{*}, 0\right) \\
& R=\left(B, D ; 0,0 ; B,-C ; 0,0 ;-B, D ; 0,0 ; A^{*}, 0\right) \\
& S=\left(0,0 ; A, C ; 0,0 ;-B,-C ; 0,0 ; A,-C ; 0,-D^{*}\right) \\
& T=\left(0,0 ; B, D ; 0,0 ; A,-D ; 0,0 ; B,-D ; 0, C^{*}\right),
\end{aligned}
$$

and

$$
\begin{aligned}
& Q=(A, C ; A, D ;-A, C) \\
& R=(B, D ; B,-C ;-B, D) \\
& S=(A, C ;-B,-C ; A,-C) \\
& T=(B, D ; A,-D ; B-D) .
\end{aligned}
$$

Similarly from $N S(5):(111-1 ; 1010-, 010-0)$, we obtain the following $R D(11 t)$.

$$
\begin{aligned}
Q= & \left(A, C ; 0,0 ;-A, D ; 0,0 ; A, C ; 0,0 ; A,-D ; 0,0 ; A,-C ; 0,0 ;-B^{*}, 0\right), \\
R= & \left(B,-D ; 0,0 ;-B, C ; 0,0 ; B, D ; 0,0 ; B,-C ; 0,0 ; B, D ; 0,0 ; A^{*}, 0\right), \\
S= & (0,0 ;-A,-C ; 0,0 ;-B,-C ; 0,0 ; A,-C ; 0,0 ; B, C ; 0,0 ; A,-C ; \\
& \left.0,-D^{*}\right) \\
T= & (0,0 ; B,-D ; 0,0 ;-A,-D ; 0,0 ; B,-D ; 0,0 ; A, D ; 0,0 ;-B,-D ; \\
& \left.0, C^{*}\right) .
\end{aligned}
$$

Also from given Golay sequences $G C S(g):(F, H)$ regarded as $N S(g)$ : $\left(F: H, 0_{g}\right)$, we obtain the following $R D((2 g+1) t)$ from Theorem 1, i.e., $2 g+$ $1=3,5,9,17,21,33,41,53,65,81, \cdots$.

$$
\begin{aligned}
& Q=\left(A f_{1}^{*}, C h_{1} ; 0,0 ; A f_{2}^{*}, C h_{2} ; 0,0 ; \cdots ; A f_{g}^{*} ; C h_{g} ; 0,0 ;-B^{*}, 0\right), \\
& R=\left(B f_{1}^{*}, D h_{1}^{*} ; 0,0 ; B f_{2}^{*}, D h_{2}^{*} ; 0,0 ; \cdots ; B f_{g}^{*}, D h_{g}^{*} ; 0,0 ; A^{*}, 0\right), \\
& S=\left(0,0 ; A h_{1}^{*},-C f_{1} ; 0,0 ; A h_{2}^{*},-C f_{2} ; 0,0 ; \cdots ; A h_{g}^{*},-C f_{g} ; 0,-D^{*}\right), \\
& T=\left(0,0 ; B h_{1},-D f_{1} ; 0,0 ; B h_{2},-D f_{2} ; 0,0 ; \cdots ; B h_{g},-D f_{g} ; 0, C^{*}\right) .
\end{aligned}
$$

Thus Theorem 1 is a generalization of [Y1, Y2, Theorem 2] and we can construct $R D(u t)$ for $u=2 n+1=15,19,23,27,31,51$ and 59 , which are not 
published before. Although sets of $N S(n)$ have not been found for $n \geq 17$ (except $n=25,29$ and $g$ ), we can construct $R D((2 n+1) t)$ for any $n$, by Theorem 1, if a new set of $N S(n)$ can be found in the future.

Theorem 3 can be regarded as a generalization of [Y2, Theorem 3]. For Example, from $N N(5):(11,1 ; 1-, 00)$ and $N N(13):(10-011,10101 ; 11-$ $1--\}$, we obtain respectively the following $R D(5 t)$ and $R D(13 t)$.

$$
\begin{aligned}
& Q=\left(-A,-C ; A,-D^{*} ; 0,-D^{*} ; 0,0 ; 0,0\right), \\
& R=\left(0,0 ; 0,0 ;-B, 0 ;-B,-C^{*} ; A^{*}, C^{*}\right), \\
& S=\left(B,-D ;-B, C^{*} ; 0, C^{*} ; 0,0 ; 0,0\right), \\
& T=\left(0,0 ; 0,0 ; A, 0 ; A, D^{*} ; B^{*},-D^{*}\right) ;
\end{aligned}
$$

and

$$
\begin{aligned}
Q= & \left(-A,-C ;-A,-D^{*} ; A,-C ;-A, D^{*} ; A,-C ; A,-D^{*} ; 0,-D^{*} ;\right. \\
& 0,0 ; 0,0 ; 0,0 ; 0,0 ; 0,0 ; 0,0), \\
R= & \left(B,-D ; B, C^{*} ;-B,-D ; B,-C^{*} ;-B,-D ;-B, C^{*} ; 0, C^{*} ; 0,0 ;\right. \\
& 0,0 ; 0,0 ; 0,0 ; 0,0 ; 0,0), \\
S= & \left(0,0 ; 0,0 ; 0,0 ; 0,0 ; 0,0 ; 0,0 ;-B, 0 ;-B,-C^{*} ; A^{*},-C^{*} ; B, C^{*} ;\right. \\
& \left.A^{*},-C^{*} ;-B, C^{*} ; A^{*}, C^{*}\right) \\
T= & \left(0,0 ; 0,0 ; 0,0 ; 0,0 ; 0,0 ; 0,0 ; A, 0 ; A, D^{*} ; B^{*}, D^{*} ;-A,-D^{*} ; B^{*}, D^{*} ;\right. \\
& \left.A,-D^{*} ; B^{*},-D^{*}\right) .
\end{aligned}
$$

We can also construct regular $\delta$-codes $R D(n t)$ by Theorem 3 in a similar way for $n=9,17,21,25,29,33,37,41,45$ and any $n$ for which a set of $N N(n)$ exists.

Finally we remark that in order to compose 4-symbol $\delta$-codes successfully by application of the Lagrange identity, when $e \neq 0$ all terms $a, b, \ldots, h$ have to be quasi-symmetric except possibly $e$, since $e^{\prime}$ is not involved in $(\mathrm{L})$ as in Theorems 1, 3 and 4; and when $e=0 f, g$ and $h$ have to be quasi-symmetric but not $a, b, c$ and $d$, since there are no $a^{\prime}, b^{\prime}, c^{\prime}$ and $d^{\prime}$ in (L) as in Theorems 2 and $2^{*}$.

\section{REFERENCES}

[A] S. S. Agaian, Hadamard matrices and their applications, Springer-Verlag, Berlin, 1985.

[GS] A. V. Geramita and J. Seberry, Orthogonal designs, Dekker, New York, 1979.

[Go] J. M. Goethals and J. J. Seidel, A skew Hadamard matrix of order 36, J. Aust. Math. Soc. 11 (1970), 343-344.

[G] M. J. E. Golay, Complementary series, IRE Trans. Information Theory, IT-7 (1961), 82-87.

[H.] M. Hall, Jr., Combinatorial theory, 2nd ed., Wiley and Sons, New York, 1986.

[HS] M. Harwit and N. J. A. Sloane, Hadamard transform optics, Academic Press, New York, 1979.

[HW] A. Hedayat and W. D. Wallis, Hadamard matrices and their applications, Ann. Math. Stat. 6 (1978), 1184-1238.

[K] C. Koukouvinos, S. Kounias and J. Seberry, Further results on base sequences, disjoint complementary sequences, $O D(4 t ; t, t, t)$ and the excess of Hadamard matrices, (to appear). 
[M] A. C. Mukhopadyay, Some infinite classes of Hadamard matrices, J. Combin. Theory (A) 25 (1978), 128-141.

[S] E. Spence, An infinite family of Williamson matrices, J. Aust. Math. Soc. (A) 24 (1977), 252256.

[T1] R. J. Turyn, Hadamard matrices, Baumert-Hall units, four symbol sequences, pulse compression, and surface wave encodings, J. Combin. Theory (A) 16 (1974), 313-333.

[T2] _ Personal communication, 1980.

[T3] _ _ An infinite class of Williamson matrices, J. Combin. Theory (A) 12 (1972), 319-321.

[W] J. Seberry Wallis, Construction of Williamson type matrices, J. Linear and Multilinear Alg. 3 (1975), 197-207.

[Wi] J. Williamson, Hadamard's determinant theorem and the sum of four squares, Duke Math. J. 11 (1944), 65-81.

[Y1] C. H. Yang, A composition theorem for $\delta$-codes, Proc. Amer. Math. Soc. 89 (1983), 375-378.

[Y2] _ Lagrange identity for polynomials and $\delta$-codes of lengths $7 t$ and $13 t$, Proc. Amer. Math. Soc. 88 (1983), 746-750.

[Y3] _ Hadamard matrices and $\delta$-codes of length $3 n$, Proc. Amer. Math. Soc. 85 (1982),

[Y4] __ Hadamard matrices, finite sequences, and polynomials defined on the unit circle, Math. Comp. 33 (1979), 688-693.

[Y5] __, On Golay sequences and near normal sequences, (to appear).

Department of Mathematical Sciences, State University of New York, Oneonta, NEW YORK 13820 